\title{
Autogestión hospitalaria en cifras
}

\author{
MIRZA RETAMAL ${ }^{(1)}$
}

En los últimos decenios, el panorama de la salud en Chile ha variado sustancialmente, debido a situaciones tales como el cambio en la composición estructural de la población ${ }^{1}$, los nuevos fenómenos epidemiológicos, la implementación de nuevos procedimientos, (con el mayor costo que implica) y el aumento de expectativas y exigencias de calidad de la atención por parte de los usuarios ${ }^{2}$. Este escenario ha llevado a las autoridades sanitarias a efectuar una profunda revisión del sistema de salud y a partir de ese análisis, establecer nuevos modelos de atención en salud ${ }^{3}$.

Con el fin de enfrentar los desafíos derivados de estos cambios, en el año 2002 el Ministerio de Salud publicó los Objetivos Sanitarios y Metas para la Década 2000-2010, que impulsan mejoras en la gestión y centran el sistema de atención de salud en las necesidades del usuario ${ }^{4}$.

Para perfeccionar los modelos de atención y gestión del sistema, se evaluaron las dificultades que los gestores hospitalarios tienen para adaptar su oferta asistencial a las nuevas necesidades y expectativas de la población -en particular en un escenario con garantías explícitas- ${ }^{5}$. En la búsqueda de mejoramiento, el año 2004 se aprobó la ley $\mathrm{N}^{\circ} 19.937$, denominada "Ley de Autoridad Sanitaria", que modifica el decreto ley $N^{\circ} 2763$ de 1979 , creando, entre otros cambios, la figura de "Establecimiento de Autogestión en Red" (EAR) $)^{6}$ Y en septiembre del año 2005 se aprobó el decreto $\mathrm{N}^{\circ} 38$ del Minsal, que establece el reglamento orgánico para este tipo de establecimientos?

En lo sustantivo, este nuevo marco normativo otorga a los hospitales espacios de mayor flexibilidad y autonomía para asignar sus recursos, comprar servicios de apoyo, vender servicios al sector privado y gestionar su presupuesto $^{8}$, a través de transferirles las facultades administrativas existentes en los servicios de salud.

El primer paso en el proceso de creación de EAR, fue la inscripción de los establecimientos interesados en la Subsecretaría de Redes Asistenciales $^{6-7}$. De un número inicial de 59 hospitales de mayor complejidad, durante el trienio 2006-2008, 11 completaron el proceso y consiguieron la calidad de EAR.

La calidad de autogestionado permitiría idear un nuevo tipo de desarrollo institucional, con líneas concretas de evolución en todas sus áreas. Esto se expresaría en diferentes maneras de encarar las necesidades y establecer las prioridades para empezar la redistribución de los recursos y la formulación de un presupuesto autónomo y para adecuar la planta a los requerimientos de las nuevas formas de trabajo del hospital. También se fortalecería la integración del establecimiento a la red asistencial, configurando un modelo que permitía la gestión de la demanda en redes de atención, bajo la denominación de "Autogestión Hospitalaria en Red"5-8-9.

La gestión de los establecimientos hospitalarios es diferente e irregular, aun en aquellos que tienen los mismos niveles de complejidad y financiamiento ${ }^{11}$. Es, entonces, que la calidad de autogestionado depende del cumplimiento de ciertos estándares que garanticen la seguridad de las prestaciones que

(1) Escuela de Salud Pública. Facultad de Medicina. Universidad de Chile. Independencia 939. Santiago. Chile. mirzaretamal@gmail.com 
otorgan, siendo responsabilidad del Ministerio de Salud la elaboración de dichos estándares y sus criterios de verificación. (Figuras 1 y 2)

\section{ANALISIS}

Con el fin de obtener una primera impresión de las características de los resultados del nuevo sistema hospitalario, procederemos a revisar

\section{Requisitos hospitales autogestionados}

a) Demostrar la existencia de una articulación adecuada con la Red Asistencial.

b) Estar registrado en la Superintendencia de Salud como prestador acreditado.

c) Tener un Plan de Desarrollo Estratégico concordante con las políticas, planes y programas de la Red Asistencial (en Recursos Humanos, Gestión Financiero-Contable y Presupuestaria, de Mejoramiento índices de satisfacción usuaria, de Gestión Clínica y Administrativa, Procedimientos de recaudación de ingresos, Plan de Actividades de Auditoria interna, Sistemas de Planificación y Control de Gestión).

d) Contar con un Plan Anual de Actividades y un Plan de Inversiones que implemente el Plan de Desarrollo Estratégico.

e) Mantener el equilibrio presupuestario y financiero.

f) Tener sistemas de medición de costos, de calidad de las atenciones prestadas, de satisfacción de los usuarios y de las metas sanitarias establecidas en Convenios o Compromisos de Gestión.

g) Cumplir con las Garantías Explícitas en Salud vigentes.

h) Tener implementado procedimientos de cobro y recaudación de ingresos.

i) Contar con mecanismos formales de participación: (Consejo Técnico, Comités, Unidades Asesoras) con reglamentaciones internas de funcionamiento y sistemas de cuenta pública a la comunidad.

j) Sistema especializado que cuente con mecanismos para el manejo de las peticiones, críticas, reclamos, sugerencias y felicitaciones, orientado a recibirlos y solucionarlos.

Figura 1. Requisitos hospitales autogestionados (Art. 16 Decreto 38/2005 de la Subsecretaría de Salud Pública del Minsal). algunos de los indicadores de la actividad hospitalaria habitualmente utilizados. Estas mediciones corresponden al año 2009 y con ellas se intentará comparar los EAR con establecimientos que no ostentaban esta calidad hasta diciembre del $2009^{10}$.

Como muestra se escogieron cinco hospitales con la calidad de autogestionados, excluyendo a los institutos de especialidades, entre ellos los hospitales pediátricos. Dentro del grupo de los no autogestionados, se eligieron recintos con una composición de servicios clínicos semejantes $\mathrm{y}$, cuando fue posible, de tamaño similar.

Para el análisis se establecieron 4 grupos, compuestos por 1 ó 2 hospitales acreditados como autogestionados en red a diciembre del 2009 y 2 hospitales no acreditados a esa fecha, agrupados de acuerdo a la diversidad de servicios clínicos -según el número de servicios clínicos de Medicina, Cirugía y de Especialidades, unidades de cuidados Intensivos de Adultos, Pediatría y Neonatología y Unidades de Tratamiento Intermedio que posean-. El Grupo 1 lo componen el Hospital Dr. Hernán Henríquez Aravena (Temuco) -autogestionado desde 2006y los hospitales Dr. Leonardo Guzmán (Antofagasta) y Regional de Rancagua. En el segundo Grupo se incluyó al Hospital Clínico Herminda Martín (Chillán) -autogestionado desde el 2008- y a los hospitales Dr. Gustavo Fricke (Viña del Mar) y Carlos Van Buren (Valparaíso). En el Grupo 3 se aglutinó al Hospital El Pino -autogestionado desde el 2008, al Hospital San Juan de Dios (La Serena) y el Hospital de San Camilo (San Felipe). Por último, se asoció al Hospital Dr. Luis Tisné y Hospital San Juan de Dios (Los Andes) -autogestionados desde el 2007 y el 2008 respectivamente- con el Hospital Dr. Antonio Tirado Lanas (Ovalle) y Hospital San José (Melipilla). (Tabla 1).

Se estudiaron los siguientes indicadores habituales de actividad hospitalaria de estos 13 centros asistenciales del año 2009 ${ }^{10-12}$ :

- Días-cama disponibles: definidos como el número de días en que las camas hospitalarias están habilitadas, en condiciones de ser utilizadas por los pacientes que se 
Los establecimientos autogestionados, como parte de la red asistencial, deberán:

a) Desarrollar el tipo de actividades asistenciales, grado de complejidad técnica y especialidades que determine el Director del Servicio.

b) Atender beneficiarios de las leyes $\mathrm{N}^{\circ} 18.469 \mathrm{y}$ $\mathrm{N}^{\mathrm{o}} 16.744$, que hayan sido referidos por alguno de los establecimientos de las Redes Asistenciales y los casos de urgencia o emergencia.

c) Mantener sistemas de información compatibles con los de la Red.

d) Entregar la información estadística, de atención de pacientes.

e) Contar con un sistema de registro y gestión de listas de espera y cumplir con las metas de reducción acordadas con el Servicio respectivo.

f) Dar cumplimiento a los convenios celebrados con el Fondo Nacional de Salud, el respectivo Secretario Regional Ministerial de Salud y con el Servicio.

Figura 2. Requisitos establecimientos como parte de la red asistencial. (Art. 18, Reglamento 38/2005 de la Subsecretaría de Salud Pública del Minsal).

hospitalicen. Se incluyen tanto aquellas que están ocupadas como las desocupadas, pero en condiciones de uso inmediato, en un período determinado.

- El número de egresos hospitalarios: Egreso se define como el retiro de un paciente que ha ocupado una cama del Hospital ya sea debido a alta, fallecimiento, traslado a otro establecimiento, retiro voluntario del paciente $\mathrm{u}$ otro. No incluye los traslados entre servicios al interior del hospital.

- El número de egresos por cama: Es un indicador de rendimiento del recurso cama, que alude al promedio de egresos que cada cama generó durante un período de tiempo.

- El promedio de días de estada es el número promedio de días de hospitalización por cada egreso en un período de tiempo dado, considerando la estancia total en el hospital, así se componga de la hospitalización en varios servicios.

- Índice Ocupacional: entendido como el número de días-cama que estuvieron ocupados durante un período, respecto del total disponibles, expresado en porcentaje.

- Tasas de Letalidad: establecida como la relación entre el número de defunciones ocurridas durante un período en un establecimiento de salud y el número de egresos del mismo período.

\section{RESULTADOS}

En el Grupo 1 la dotación de camas es ligeramente mayor en el Hospital de Antofagasta mientras que el número de días-cama disponibles es inferior al del Hospital Hernán Henríquez Aravena de Temuco. Esto podría explicarse porque el número de camas disponibles puede variar diariamente debido a que se agregan camas por demanda estacional o emergencia y que se retiren camas para reparación, desinfección, mantención ${ }^{13}$, etc. El índice ocupacional promedio es mayor a 80 en los 3 recintos y cercano a 90 en Hospital de Temuco, lo que implica que existirían cifras superiores en ciertos períodos del año, lo que representa una cuestión difícil de administrar. El promedio de días de estada es mayor en el Hospital de Temuco, pero también el número de egresos por cama. La tasa de letalidad muy superior en el Hospital de Rancagua.

En el Grupo 2, los días camas disponibles son similares en el Hospital de Chillán y Valparaíso y mayores que el de Viña del Mar. La cantidad de egresos y egresos por cama es mayor en el Van Buren, con un índice de ocupación similar entre los 3 centros y un promedio de días de estada y una tasa de letalidad menores en el de Valparaíso.

En el Grupo 3 se aprecia que el Hospital El Pino y de San Camilo presentan similar número de días-cama disponibles, con un menor número de egresos y egresos por cama en este último, lo que se asocia a un promedios de días de estada ligeramente mayores. Este mismo Hospital revela un índice ocupacional de 73,9\%. Lo que, unido a lo anterior, sugiere una menor demanda de la población respecto de la capacidad instalada. En este grupo, la performance del Hospital San Juan de Dios de 
Tabla 1. Indicadores de Actividad Hospitalaria 2009 en Grupos de Hospitales acreditados con Establecimientos Autogestionados en Red y Hospitales No Autogestionados al 31 de Diciembre del 2009.

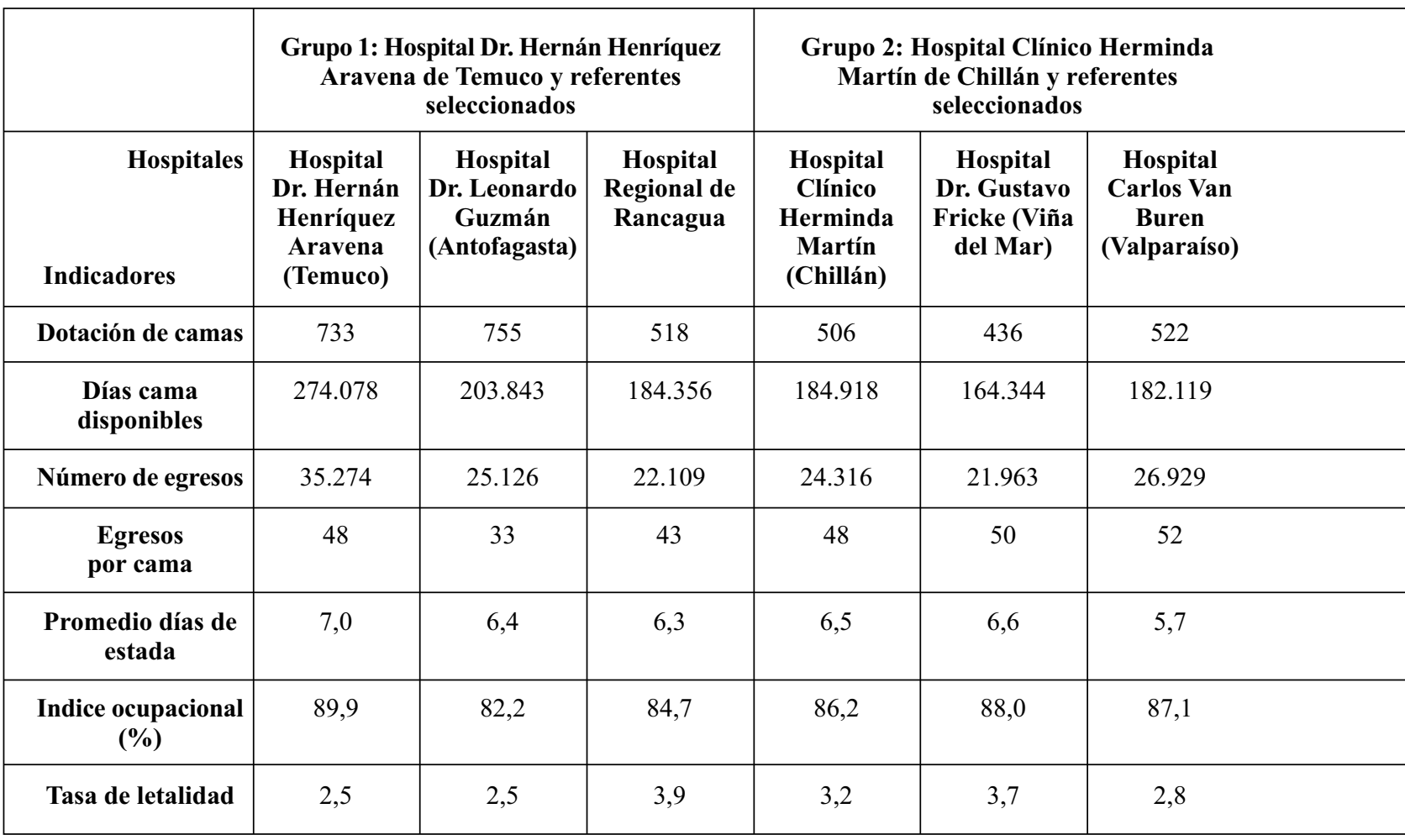

Fuente: DEIS Minsal

La Serena tiene un mejor perfil. Más egresos, más egresos por cama, menor promedio de estadía, niveles de ocupación cercanos al $80 \%$.

El Grupo 4 donde se asocian 2 hospitales acreditados como autogestionados en red hasta el 2009 y 2 sin esa calidad a esa misma fecha, con similar número de servicios, (El Hospital Dr. Luis Tisné, cuenta además con UCI) y con diferente número de camas, llama la atención un significativo menor número de días-cama disponibles en el Hospital San Juan de Dios de Los Andes en relación a sus dotación de camas, con un número de egresos por cama también inferior, a consecuencia de lo mismo. Por alguna razón que no fue posible conocer, el Hospital parece haber estado con una proporción de camas en trabajo bastante menor a las camas de dotación durante el año 2009. Entre los 4 el promedio de días de Estada es similar, con un índice ocupacional inferior o cercano al $75 \%$ en 3 de ellos. Las tasas de letalidad no varían significativamente.

\section{CONCLUSIÓN}

La comparación de indicadores de uso habitual no presenta diferencias importantes entre hospitales autogestionados y no autogestionados. No existe algún patrón observado que permita levantar una hipótesis razonable relativa a diferencias en la performance de los mismos, vista ésta a través de los indicadores gruesos habituales. Pero, cuidado, lo anterior no implica que la autogestión no haya tenido efecto alguno sobre el desempeño hospitalario. Estos resultados parecen (o deberían estar) afectados por cuestiones estructurales como desbalances entre la capacidad instalada y la demanda, en cualquier sentido, lo que es válido para cualquiera de los hospitales y dice 
Grupo 3: Hospital El Pino de San Bernardo y referentes seleccionados

Grupo 4: Hospitales Dr. Luis Tisné de Peñalolén y San Juan de Dios de Los Andes y referentes seleccionados

\begin{tabular}{|c|c|c|c|c|c|c|}
$\begin{array}{c}\text { Hospital } \\
\text { EI Pino }\end{array}$ & $\begin{array}{c}\text { Hospital San } \\
\text { Juan de Dios } \\
\text { (La Serena) }\end{array}$ & $\begin{array}{c}\text { Hospital de } \\
\text { San Camilo } \\
\text { (San Felipe) }\end{array}$ & $\begin{array}{c}\text { Hospital } \\
\text { Dr. Luis Tisné }\end{array}$ & $\begin{array}{c}\text { Hospital San } \\
\text { Juan de Dios } \\
\text { (Los Andes) }\end{array}$ & $\begin{array}{c}\text { Hospital } \\
\text { Dr. Antonio } \\
\text { Tirado Lana } \\
\text { (Ovalle) }\end{array}$ & $\begin{array}{c}\text { Hospital } \\
\text { San José } \\
\text { (Melipilla) }\end{array}$ \\
\hline 283 & 282 & 238 & 340 & 214 & 192 & 161 \\
\hline 83.498 & 92.481 & 84.878 & 116.418 & 44.685 & 69.882 & 46.669 \\
\hline 14.188 & 15.418 & 10.866 & 21.227 & 7.635 & 10.794 & 7.782 \\
\hline 50 & 55 & 46 & 62 & 36 & 56 & 48 \\
\hline 5,0 & 4,8 & 5,3 & 4,5 & 4,5 & 4,7 & 4,3 \\
\hline 3,1 & 81,6 & 73,9 & 82,3 & 74,8 & 75,9 & 73,9 \\
\hline
\end{tabular}

relación con cuestiones difíciles de modificar en el corto plazo (inversiones mediante). En la práctica, estos son indicadores "gruesos".

Una cuestión de la mayor importancia, sin embargo, dice relación con los elevados niveles de ocupación que se observan, los que tratándose de promedios, en algunos momentos del año deben estar ocasionando problemas operacionales importantes y agudizando el déficit de oferta en algunos servicios clínicos, en particular en aquellos que deberían contar con más holgura, por la trascendencia de un rechazo, como son las maternidades y los servicios de cuidados intermedios e intensivos. Pero tampoco en esto último es posible observar un punto que permita establecer diferencias entre hospitales autogestionados y no autogestionados.

Habida cuenta de que lo incipiente del proceso también podría ser una razón que explique la no verificación de cambios importantes a la fecha, se debe tener también en cuenta que los primeros 11 hospitales autogestionados fueron calificados como tal precisamente porque cumplían antes ciertos niveles de exigencia que se reflejan en una serie de indicadores. En consecuencia, es posible establecer una hipótesis en cuanto a que los resultados de la autogestión sí deberían estar reflejándose en indicadores más finos, que den respuesta específica a los requerimientos establecidos en las Figuras 1 y 2. En ese caso podría tomarse un grupo de ellos que fueron relevantes para la certificación y ver cómo se han modificado en el tiempo. Considerando la disponibilidad de información, sólo una investigación operacional ad-hoc permitiría corroborar o descartar esta hipótesis. Una segunda línea de trabajo puede desarrollarse en torno a los problemas estructurales de los hospitales frente al escenario cambiante descrito 
al inicio de este documento. En efecto, las inversiones y ajustes tecnológicos de la oferta de servicios parecen correr siempre detrás de la realidad con bastante rezago, al tiempo de transformarse en uno de los problemas universales del sector salud en la actualidad ${ }^{14}$.

\section{REFERENCIAS}

1. REV ESP SALUD PÚBLICA. La transición demográfico-epidemiológica en Chile, 1960-2001. 2003; 77: 605-13.

2. REV. ESP. SALUD PUBLICA Reflexión sobre la planificación de los recursos humanos y la autonomía de gestión en los hospitales de Chile. Vol.83 no.3 Madrid May\June 2009.

3. MINISTERIO DE SALUD. Chile. Modelo de atención integral de salud. Serie Cuadernos $N^{\circ} 1$. Marzo de 2006

4. MINISTERIO DE SALUD. Los Objetivos Sanitarios para la Década 2000-2010. Santiago de Chile: Ministerio; 2002.

5. CUADERNOS MEDICO SOCIALES Los desafíos pendientes de la autogestión hospitalaria en red.
Sociedad Chilena de Administradores en Atención Médica y Hospitalaria. Serie.2009

6. MINISTERIO DE SALUD de Chile. Ley $\mathrm{N}^{\circ} 19.937$ de Autoridad Sanitaria y Gestión. Chile.

7. MINISTERIO DE SALUD. Subsecretaría de Salud Pública. Reglamento orgánico de los establecimientos de salud de menor complejidad y de los establecimientos de autogestión en red. Decreto $\mathrm{N}^{\circ}$ 38 del 2005.

8. REV. CHIL. PEDIATR. Los desafíos de la autogestión hospitalaria 2008; 79 (2): 127-130.

9. EXPANSIVA. Serie En Foco. Separación de funciones en la reforma del sector salud en Chile: Tres Visiones 2007.

10. DEIS MINSAL. Estadísticas Hospitalarias 2009.

11. REV MÉD CHILE. Indicadores de gestión de servicios de salud públicos y asignación de recursos desde el Ministerio de Salud de Chile. 2004; 132: 1532-1542

12. INE. Instituto Nacional de Estadísticas.

13. ERNESTO MEDINA LOIS ANA MARÍA KAEMPFFER R. Elementos de Salud Pública . Cap 3. Biblioteca Digital Universidad de Chile.

14. RECHEL, B Y OTROS. European Observatory of Health Systems and Policies: Capital Investment for Health, Case studies from Europe. Observatory Studies Series No18. 2009.

Usted puede comentar éste y otros artículos publicados en la Revista Chilena de Salud Pública, enviando un correo electrónico a revistasp@med.uchile.cl 